Recepción: 20 / 04 / 2017

Aceptación: 20 / 05 / 2017

Publicación: 15 / 06 / 2017

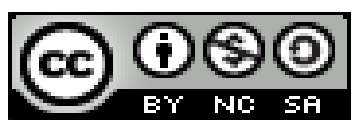

Ciencias de la Salud

Artículo de Investigación

\title{
Prevalencia de sobrepeso y obesidad
}

\section{Prevalence of overweight and obesity}

\section{Prevalência de sobrepeso e obesidade}

M.Sc. Josefina E. Ramírez-Amaya ${ }^{\text {I }}$ josefina.ramireza@ug.edu.ec

\section{M.Sc Fanny P. Cabrera-Jiménez II} fanny.cabreraj@ug.edu.ec

M.Sc. Pedro L. Maldonado-Álava III pedro.maldonadoa@ug.edu.ec

M.Sc. Julio J. De la Torre-Chávez IV juliodelatorrec@hotmail.com

Correspondencia: josefina.ramireza@ug.edu.ec

I. Master en salud pública, Especialista en docencia superior, Doctora en medicina y cirugía. Docente Universidad de Guayaquil, Guayaquil, Ecuador.

II. Master en Bioquímica clínica, Especialista en docencia superior, Doctora en medicina y cirugía. Docente Universidad de Guayaquil, Guayaquil, Ecuador.

III. Master en salud pública, Especialista en docencia superior, Doctora en medicina y cirugía. Docente Universidad de Guayaquil, Guayaquil, Ecuador.

Iv. Master en Gerencia Educativa, Especialista en Gestión de Procesos Educativos, Diplomado en Docencia Superior, Docente Universidad de Guayaquil, Guayaquil, Ecuador. 


\section{Resumen}

Se definen como una acumulación anormal o excesiva de grasa que puede ser perjudicial para la salud, La obesidad y el sobrepeso es un problema de salud creciente que afecta cada vez más a niños en el mundo sin interesar género, grupo étnico o nivel socioeconómico, es una enfermedad crónica, compleja y multifactorial suele iniciarse en la infancia y la adolescencia, se establece por un desequilibrio entre la ingesta y el gasto energético.se involucran factores genéticos y ambientales, que determinan un trastorno metabólico que conduce a una excesiva acumulación de grasa corporal para el valor esperado según el sexo, talla, y edad.

Se realizó un estudio transversal, descriptivo, observacional y comparativo; Se entrevistó de forma directa tipo encuestas a alumnos de la escuela de educación básica Héctor Lara Zambrano en la ciudad de milagro, a fin de identificar los factores de riesgo de sobrepeso y obesidad.

La prevalencia de sobrepeso (12\%) y obesidad (12\%) fue más alta en la población masculina que en la femenina (52\%) sobrepeso y (64\%) obesidad. Los niños de 7-8 años presentaron una prevalencia de $15 \%$ de sobrepeso, y $18 \%$ de obesidad.

Palabras claves: Grasa; obesidad; salud; infancia; adolescencia. 


\section{Abstract}

They are defined as an abnormal or excessive accumulation of fat that can be detrimental to health. Obesity and overweight is a growing health problem that increasingly affects children around the world without interest in gender, ethnic group or socioeconomic status. A chronic, complex and multifactorial disease usually started in childhood and adolescence, is established by an imbalance between intake and energy expenditure. Genetic and environmental factors are involved, which determine a metabolic disorder that leads to excessive accumulation of body fat For the expected value according to sex, height, and age.

A cross-sectional, descriptive, observational and comparative study was performed; We directly interviewed students from the Héctor Lara Zambrano basic education school in the city of miracle, in order to identify risk factors for overweight and obesity.

The prevalence of overweight (12\%) and obesity (12\%) was higher in the male population than in the female population (52\%) and overweight (64\%). Children aged 7-8 years had a prevalence of $15 \%$ of overweight, and $18 \%$ of obesity.

Key words: Fat; obesity; health; childhood; adolescence. 


\section{Resumo}

É definida como o acúmulo de gordura anormal ou excessivo que pode ser prejudicial à saúde, obesidade e excesso de peso é um problema de saúde crescente que afecta cada vez mais crianças no mundo sem sexo interesse, etnia ou condição socioeconômica é uma doença crónica, complexa e multifactorial geralmente inicia na infância e adolescência é estabelecida por um desequilíbrio entre a ingestão e despesas energético.se envolvem factores genéticos e ambientais que determinam uma doença metabólica que conduz à acumulação excessiva de gordura corporal para o valor esperado pelo sexo, tamanho e idade.

Em corte, descritiva, observação e estudo comparativo; Met digitar diretamente os alunos inquéritos realizados nas escolas do ensino básico Héctor Zambrano na cidade Lara miracle, a fim de identificar fatores de risco para sobrepeso e obesidade.

A prevalência de excesso de peso (12\%) e obesidade (12\%) foi maior entre os homens do que do sexo feminino (52\%) e com excesso de peso (64\%) de obesidade. Crianças 7-8 anos tinha uma prevalência de $15 \%$ com sobrepeso e $18 \%$ obesos.

Palavras-chave: Gordura; obesidade; saúde; infancia; adolescência. 


\section{Introducción.}

El sobrepeso y la obesidad se definen como una acumulación anormal o excesiva de grasa que puede ser perjudicial para la salud. Actualmente, el sobrepeso es una condición común y frecuente, dónde los suministros de alimentos son abundantes y predomina el sedentarismo, es un problema que preocupa a todo el mundo.

En el presente trabajo se expone algunos de los factores que causan la obesidad infantil en nuestro país, observando que el sector que la padece con mayor intensidad es la niñez en la cual han aumentado de manera significativa la obesidad y el sobrepeso.

En 2013, más de 42 millones de niños menores de cinco años de edad tenían sobrepeso. Si bien el sobrepeso y la obesidad tiempo atrás eran considerados un problema propio de los países de ingresos altos, actualmente ambos trastornos están aumentando en los países de ingresos bajos y medianos, en particular en los entornos urbanos.

En los países en desarrollo con economías emergentes (clasificados por el Banco Mundial en países de ingresos bajos y medianos) el incremento porcentual del sobrepeso y la obesidad en los niños ha sido un 30\% superior al de los países desarrollados. (OMS, 2015)

En Europa 1 de cada 6 niños o su equivalente el 20\% tiene sobrepeso, mientras que 1 de cada 20 adolescentes (el 5\%) es obeso. En España, 4 hijos de cada 10 familia (42,7\%) con edades de entre 6 a 10 años tienen sobrepeso y 1 de ellos ya es obeso. (Cordero, 2012)

La Asociación Internacional para el Estudio de la Obesidad de la prevalencia de sobrepeso (incluyendo obesidad) en la OCDE y los países emergentes entre los niños en edad escolar de entre 5 a 17 años, basado en los últimos estudios nacionales disponibles que la altura medida y peso, y el 
Josefina E. Ramírez-Amaya; Fanny P. Cabrera-Jiménez; Pedro L. Maldonado-Álava; Julio J. De la Torre-Chávez

uso de las definiciones IASO de sobrepeso / obesidad. Uno de cada cinco niños se ven afectados por el exceso de peso corporal en todos los países, y en Grecia, Estados Unidos e Italia la cifra está más cerca de un tercio. Sólo en China, Corea y Turquía son $10 \%$ o menos de los niños con sobrepeso.

En la mayoría de los países, los niños tienen mayores tasas de sobrepeso y obesidad que hacen las niñas. Las niñas tienden a tener tasas más altas en los países nórdicos (Suecia, Noruega, Dinamarca), así como en el Reino Unido, los Países Bajos y Australia. (OECD, 2011)

En América Latina y el Caribe, se ha notificado un aumento notable en la prevalencia de exceso de peso. En los Estados Unidos y Europa se observan las tasas más elevadas de sobrepeso y obesidad, pero la tendencia de incremento es generalizada en países tan diversos como Brasil y China.

La obesidad y el sobrepeso han sido prácticamente estables o han crecido modestamente, en Canadá, Inglaterra, Italia, Corea, España y Estados Unidos, pero se han incrementado en un 2.3\% más en Australia, Francia, México y Suiza. La crisis económica es probable que hayan contribuido a un mayor crecimiento de la obesidad. (oecd.org, 2014)

En México, las tasas de sobrepeso infantil se encuentran entre las más altas del área de la OCDE. Datos internacionales recolectados por la Asociación Internacional para el Estudio de la Obesidad muestran que casi 1 de cada 3 niños padece sobrepeso en México, en comparación con el 23\% de los niños y el $21 \%$ de las niñas, en promedio, en los países de la OCDE. (oecd.org, 2014)

En la niñez, la interacción entre factores genéticos, biológicos, psicológicos, socioculturales y medioambientales es evidente. (Morales, 2013) 
En Ecuador el $15 \%$ de la población escolar presenta retardo en talla y va acompañada de un aumento de la prevalencia de sobrepeso y obesidad (29.9\%), en la edad preescolar es de $8.5 \%$ y se triplica a pasar a la edad escolar. (Ministerio de Salud Pública, Instituto Nacional de Estadísticas y Censos, 2013)

En su origen se involucran factores genéticos y ambientales, que determinan un trastorno metabólico que conduce a una excesiva acumulación de grasa corporal para el valor esperado según el sexo, talla, y edad.

La obesidad infantil ha sido definida considerando la relación entre el peso total y la talla estimada mediante el índice de masa corporal $(\mathrm{IMC}=$ peso en $\mathrm{kg} /$ talla en $\mathrm{m} 2)$. El sobrepeso infantil se establece a partir del centil 75 en las curvas de IMC y la obesidad infantil a partir del centil 85.

El 10\% de los niños del mundo en edad escolar tienen un exceso de grasa corporal con un riesgo aumentado de desarrollar enfermedades crónicas. Un cuarto de estos niños tienen obesidad y algunos tienen múltiples factores de riesgo para desarrollar diabetes tipo 2, enfermedad cardíaca y otras comorbilidades antes o durante la adultez temprana. (Gilardon, 2013)

También se han descrito problemas ortopédicos, trastornos en la respuesta inmune con aumento en la susceptibilidad a infecciones y alteraciones cutáneas.

Las consecuencias psicosociales de la distorsión de la imagen física para el niño obeso pueden ser tan importantes e incluso más que las físicas. La baja autoestima, aislamiento social, discriminación y patrones anormales de conducta son algunas consecuencias frecuentes.

En su etiología interviene diferentes factores; en países industrializados el problema refleja condiciones socioeconómicas relacionadas con el exceso en el consumo de alimentos y hábitos de 
vida inadecuados; en los países menos desarrollados se debe a la existencia de consumo distorsionado debido a la necesidad de llenar los requerimientos con calorías de bajo costo provenientes de los carbohidratos y grasas saturadas.

La obesidad es una enfermedad crónica de origen multifactorial caracterizada por la acumulación excesiva de grasa o hipertrofia general del tejido adiposo en el cuerpo; es decir, cuando la reserva natural de energía de los humanos, almacenada en forma de grasa corporal se incrementa hasta un punto donde está asociada con numerosas complicaciones, condiciones de salud o enfermedades, e incluso con un incremento de la mortalidad.

La Organización Mundial de la Salud (OMS) define como sobrepeso cuando el índice de masa corporal (cálculo entre la estatura y el peso del individuo) es igual o mayor de $25 \mathrm{~kg} / \mathrm{m}^{2}$; y como obesidad cuando el índice de masa corporal es igual o superior a $30 \mathrm{~kg} / \mathrm{m}^{2}$; o cuando existe un perímetro abdominal aumentado en hombres mayor o igual a $102 \mathrm{~cm}$ y en mujeres mayor o igual a 88 $\mathrm{cm}$.

La obesidad y el sobrepeso infantil se están convirtiendo en uno de los problemas de salud de mayor preocupación. La gravedad del problema ha influido de manera relevante en el inicio de medidas a nivel preventivo y en la propuesta de formas innovadoras de enfocar el problema, adaptadas a la población actual y a las principales causas de aparición.

Adicionalmente, estos pacientes presentan alteraciones psicológicas y sociales, a menudo relacionadas con la disminución en la autoestima y en la autoconfianza producida por los comportamientos de una sociedad autodestructiva con el hombre. 


\section{Materiales y métodos.}

\section{Caracterización de la zona de trabajo}

Él estudió que se realizara es de tipo transversal, descriptivo, observacional y comparativo en la Escuela de educación básica Héctor Lara Zambrano situado en la ciudad de Milagro en las calles Carlos Chiriguaya y Coronel y Luis Vargas Torres.

\section{Universo y muestra}

\section{Universo}

Todos los alumnos de 3 a 12 años que asisten regularmente de una población total de 700 niños registrados de cada grado de educación inicial a séptimo año, pues representarán la totalidad de la población que reunirán los criterios de inclusión.

Se excluirán todos los niños que no cumplan con el rango de edad establecido, así como también los niños no regulares.

\section{Muestra}

Se elegirán a los niños de cada grado de la escuela básica Héctor Lara Zambrano; desde educación inicial hasta séptimo año.

\section{Viabilidad}

Este trabajo de titulación es un estudio viable porque tiene la aprobación de la Directora Lcda. María Elena Moran y de los docentes de cada grado, que permitió el acceso de cada una de la lista de los nombres de los alumnos de la institución de la Escuela Básica Héctor Lara Zambrano y el apoyo de cada uno de los padres de familia.

\section{Tipo de investigación}

Transversal; Descriptivo; Comparativo; Observacional. 
Metodología para el análisis de los resultados

Para el análisis de los resultados se realizó en hojas de cálculo del programa de Microsoft Excel, donde todos los datos se expresaron como frecuencia absoluta y porcentaje. Posteriormente la información fue ingresada en el programa estadístico IBM SPSS 19.0, la información esta presentada en forma de tablas y gráficos para una mejor comprensión, de acuerdo a las variables de estudio. Se utilizó estadística descriptiva y pruebas no paramétricas para el análisis de los datos.

\section{Resultados.}

La presente investigación ha sido planteada con el objetivo de realizar la identificación de la incidencia de sobrepeso y obesidad en 700 niños de 3 a 12 años en la escuela de educación básica Héctor Lara Zambrano en el periodo 2014 a 2015 en la ciudad de milagro.

Obtenida la información por medio de la encuesta y medición de cada alumno del peso, talla, e IMC se procedió al recuento, clasificación, tabulación y representación gráfica, proceso que permitió conocer los resultados de la investigación de campo. La información recopilada hemos resumido en tablas y gráficos.

En función de los objetivos y de la hipótesis se procede al análisis e interpretación de resultados. En la interpretación de resultados se pretende encontrar y relacionar la información recopilada con la teoría.

1. Distribución de los 700 niños de 3 a 4 años en la escuela de educación básica Héctor Lara Zambrano en el periodo 2014 a 2015 en la ciudad de Milagro según: Grupo etario.

\begin{tabular}{|l|c|c|}
\hline $\begin{array}{l}\text { ESCUELA DE EDUCACION BASICA HÉCTOR LARA } \\
\text { ZAMBRANO }\end{array}$ & Frecuencia & Porcentaje \\
\hline OBESOS & 7 & $8 \%$ \\
\hline SOBRE PESO & 6 & $7 \%$ \\
\hline NORMAL & 75 & $85 \%$ \\
\hline TOTAL & 88 & $100 \%$ \\
\hline
\end{tabular}

Tabla 1

Fuente: Escuela Héctor Lara Zambrano

Interpretación: Del total de niños afectado del grupo etario de 3 a 4 años (88 niños) el $85 \%$ peso normal, el $8 \%$ es obeso y el $7 \%$ con sobrepeso 
2. Distribución de los 700 niños de 5 a 6 años en la escuela de educación básica Héctor Lara Zambrano en el periodo 2014 a 2015 en la ciudad de Milagro según: Grupo etario.

\begin{tabular}{|l|c|c|}
\hline $\begin{array}{l}\text { ESCUELA DE EDUCACION BASICA HÉCTOR LARA } \\
\text { ZAMBRANO }\end{array}$ & Frecuencia & Porcentaje \\
\hline OBESOS & 28 & $17 \%$ \\
\hline SOBRE PESO & 18 & $11 \%$ \\
\hline NORMAL & 123 & $73 \%$ \\
\hline TOTAL & 169 & $100 \%$ \\
\hline
\end{tabular}

\section{Tabla 2}

\section{Fuente: Escuela Héctor Lara Zambrano}

Interpretación: Del total de niños afectado del grupo etario de 5 a 6 años (169 niños) el $73 \%$ peso normal, el $16 \%$ es obeso y el $11 \%$ con sobrepeso

Tabla 3. Distribución de los 700 niños de 7 a 8 años en la escuela de educación básica Héctor Lara Zambrano en el periodo 2014 a 2015 en la ciudad de Milagro según: Grupo etario.

\begin{tabular}{|l|c|c|}
\hline ESCUELA DE EDUCACION BASICA HÉCTOR LARA & Frecuencia & Porcentaje \\
ZAMBRANO & 9 & $5 \%$ \\
\hline OBESOS & 36 & $19 \%$ \\
\hline SOBRE PESO & 134 & $71 \%$ \\
\hline NORMAL & 188 & $100 \%$ \\
\hline TOTAL & \\
\hline
\end{tabular}

\section{Tabla 3}

Fuente: Escuela Héctor Lara Zambrano

Interpretación: Del total de niños afectado del grupo etario de 7 a 8 años (188 niños) el $75 \%$ peso normal, el $5 \%$ es obeso y el $20 \%$ con sobrepeso

Tabla 4. Distribución de los 700 niños de 9 a 10 años en la escuela de educación básica Héctor Lara Zambrano en el periodo 2014 a 2015 en la ciudad de Milagro según: Grupo etario.

\begin{tabular}{|l|c|c|}
\hline ESCUELA DE EDUCACION BASICA HÉCTOR LARA & Frecuencia & Porcentaje \\
ZAMBRANO & 29 & $18 \%$ \\
\hline OBESOS & 25 & $15 \%$ \\
\hline SOBRE PESO & 111 & $67 \%$ \\
\hline NORMAL & 165 & $100 \%$ \\
\hline TOTAL & \\
\hline
\end{tabular}

\section{Tabla 4}

\section{Fuente: Escuela Héctor Lara Zambrano}

Interpretación: Del total de niños afectado del grupo etario de 9 a 10 años (165 niños) el 67 $\%$ peso normal, el $18 \%$ es obeso y el $15 \%$ con sobrepeso 
Josefina E. Ramírez-Amaya; Fanny P. Cabrera-Jiménez; Pedro L. Maldonado-Álava; Julio J. De la Torre-Chávez

Tabla 5. Distribución de los 700 niños de 11 a 12 años en la escuela de educación básica Héctor Lara Zambrano en el periodo 2014 a 2015 en la ciudad de Milagro según: Grupo etario.

\begin{tabular}{|l|r|r}
\hline $\begin{array}{l}\text { ESCUELA DE EDUCACION BASICA HECTOR LARA } \\
\text { ZAMBRANO }\end{array}$ & \\
\hline & & \\
\hline OBESOS & 15 & $17 \%$ \\
\hline SOBRE PESO & 12 & $13 \%$ \\
\hline NORMAL & 63 & $70 \%$ \\
\hline TOTAL & 90 & $100 \%$ \\
\hline
\end{tabular}

\section{Tabla 5}

Fuente: Escuela Héctor Lara Zambrano

Interpretación: Del total de niños afectado del grupo etario de 11 a 12 años (90 niños) el 70 $\%$ peso normal, el $17 \%$ es obeso y el $13 \%$ con sobrepeso

Tabla 6. Distribución de los 700 niños en la escuela de educación básica Héctor Lara Zambrano en el periodo 2014 a 2015 en la ciudad de Milagro según: Tabla General

\begin{tabular}{|l|c|c|}
\hline \multicolumn{1}{|c|}{ TABLA GENERAL } & & \\
\hline ESCUELA DE EDUCACION BASICA HÉCTOR LARA & & \\
ZAMBRANO & Frecuencia & Porcentaje \\
\hline OBESOS & 83 & $12 \%$ \\
\hline SOBRE PESO & 87 & $12 \%$ \\
\hline NORMAL & 530 & $76 \%$ \\
\hline TOTAL & 700 & $100 \%$ \\
\hline
\end{tabular}

\section{Tabla 6}

\section{Fuente: Escuela Héctor Lara Zambrano}

Interpretación: Del Total de niños afectado (700 niños) el $76 \%$ peso normal, el $12 \%$ es obeso y el $12 \%$ con sobrepeso

Tabla 6. Distribución de la Total Obesidad en los 87 niños de la escuela de educación básica Héctor Lara Zambrano en el periodo 2014 a 2015 en la ciudad de Milagro según: Tabla General Obesidad

\begin{tabular}{|l|c|c|}
\hline Item & obesidad & Porcentaje \\
\hline hombres & 57 & $66 \%$ \\
\hline mujeres & 30 & $34 \%$ \\
\hline total & 87 & $100 \%$ \\
\hline
\end{tabular}

Tabla 7

Fuente: Escuela Héctor Lara Zambrano

Interpretación: Del Total de Obesidad (87 niños) el 66\% hombres, el 34 \% mujeres 
Tabla 6. Distribución del Sobrepeso 97 niño en la escuela de educación básica Héctor Lara Zambrano en el periodo 2014 a 2015 en la ciudad de Milagro según:

Tabla General Sobrepeso

\begin{tabular}{|l|c|c|}
\hline Item & Sobrepeso & Porcentaje \\
\hline hombres & 51 & $53 \%$ \\
\hline mujeres & 46 & $47 \%$ \\
\hline total & 97 & $100 \%$ \\
\hline
\end{tabular}

Tabla 8

Fuente: Escuela Héctor Lara Zambrano

Interpretación: Del Total de Sobrepeso (97 niños) el 53\% hombres, el 47\% mujeres

Encuesta aplicada a los niños afectados

1.- Familiares directos con sobrepeso y obesidad

\begin{tabular}{|l|c|c|}
\hline Respuesta & Cantidad & Porcentaje \\
\hline SI & 110 & $60 \%$ \\
\hline NO & 74 & $40 \%$ \\
\hline Total & 184 & $100 \%$ \\
\hline
\end{tabular}

Tabla 9

Fuente: Escuela Héctor Lara Zambrano

Interpretación: Del total de niños afectado del (184 niños) tienen familiares directos con sobrepeso y obesidad el $60 \% \mathrm{Si}$, el $40 \%$ No

2.- ¿Consume refrescos?

\begin{tabular}{|l|c|c|}
\hline Respuesta & Cantidad & Porcentaje \\
\hline SI & 93 & $51 \%$ \\
\hline NO & 91 & $49 \%$ \\
\hline Total & 184 & $100 \%$ \\
\hline
\end{tabular}

Tabla 10

Fuente: Escuela Héctor Lara Zambrano

Interpretación: Del total de niños afectado del (184 niños) consume refresco el $51 \%$ Si, el $49 \%$ No. 
3.- ¿Con que frecuencia los consume refresco?

\begin{tabular}{|l|c|c|}
\hline Respuesta & Cantidad & Porcentaje \\
\hline Siempre & 38 & $41 \%$ \\
\hline Casi siempre & 25 & $27 \%$ \\
\hline A veces & 18 & $19 \%$ \\
\hline Pocas veces & 12 & $13 \%$ \\
\hline Total & 93 & $100 \%$ \\
\hline
\end{tabular}

Tabla 11

Fuente: Escuela Héctor Lara Zambrano

Interpretación: Del total de niños afectado del (93 niños) con qué frecuencia consume refresco el $41 \%$ siempre, $27 \%$ casi siempre, $19 \%$ a veces, $13 \%$ pocas veces.

4.- ¿Consume golosinas?

\begin{tabular}{|l|c|c|}
\hline Respuesta & Cantidad & Porcentaje \\
\hline SI & 150 & $82 \%$ \\
\hline NO & 34 & $18 \%$ \\
\hline Total & 184 & $100 \%$ \\
\hline
\end{tabular}

Tabla 12

Fuente: Escuela Héctor Lara Zambrano

Interpretación: Del total de niños afectado del (184 niños) con sume golosinas el 82\% Si, el $18 \%$ No

5.- ¿Con que frecuencia los consume golosinas?

\begin{tabular}{|l|c|c|}
\hline Respuesta & Cantidad & Porcentaje \\
\hline Siempre & 84 & $56 \%$ \\
\hline Casi siempre & 40 & $27 \%$ \\
\hline A veces & 16 & $11 \%$ \\
\hline Pocas veces & 10 & $7 \%$ \\
\hline Total & 150 & $100 \%$ \\
\hline
\end{tabular}

Tabla 13

Fuente: Escuela Héctor Lara Zambrano

Interpretación: Del total de niños afectado del (150 niños) con qué frecuencia consume golosinas el $56 \%$ siempre, $27 \%$ casi siempre, $11 \%$ a veces, $7 \%$ pocas veces. 
6.- ¿Consume comida Chatarra; hamburguesa, hot dog, papa fritas?

\begin{tabular}{|l|c|c|}
\hline Respuesta & Cantidad & Porcentaje \\
\hline SI & 168 & $91 \%$ \\
\hline NO & 16 & $9 \%$ \\
\hline Total & 184 & $100 \%$ \\
\hline
\end{tabular}

\section{Tabla 14}

Fuente: Escuela Héctor Lara Zambrano

Interpretación: Del total de niños afectado del (184 niños) consume comida chatarra el 91\% Si, el $9 \%$ No

7.- Con qué frecuencia consume comida chatarra

\begin{tabular}{|l|c|c|}
\hline Respuesta & Cantidad & Porcentaje \\
\hline Siempre & 89 & $53 \%$ \\
\hline Casi siempre & 57 & $34 \%$ \\
\hline A veces & 17 & $10 \%$ \\
\hline Pocas veces & 5 & $3 \%$ \\
\hline Total & 168 & $100 \%$ \\
\hline
\end{tabular}

Tabla 15

\section{Fuente: Escuela Héctor Lara Zambrano}

Interpretación: Del total de niños afectado del (168 niños) con qué frecuencia consume comida chatarra el $53 \%$ siempre, $34 \%$ casi siempre, $10 \%$ a veces, $3 \%$ pocas veces.

8.- ¿Consume Frutas?

\begin{tabular}{|l|c|c|}
\hline Respuesta & Cantidad & Porcentaje \\
\hline SI & 67 & $36 \%$ \\
\hline NO & 117 & $64 \%$ \\
\hline & 184 & $100 \%$ \\
\hline
\end{tabular}

\section{Tabla 16}

Fuente: Escuela Héctor Lara Zambrano

Interpretación: Del total de niños afectado del (184 niños) consume fruta el $36 \%$ Si, el 34\% No 9.- ¿Con qué frecuencia los consume frutas?

\begin{tabular}{|l|c|c|}
\hline Respuesta & Cantidad & Porcentaje \\
\hline Siempre & 24 & $36 \%$ \\
\hline Casi siempre & 21 & $31 \%$ \\
\hline A veces & 17 & $25 \%$ \\
\hline Pocas veces & 5 & $7 \%$ \\
\hline Total & 67 & $100 \%$ \\
\hline
\end{tabular}

Tabla 17

Fuente: Escuela Héctor Lara Zambrano 
Interpretación: Del total de niños afectado del (67 niños) con qué frecuencia consume frutas el $36 \%$ siempre, $31 \%$ casi siempre, $25 \%$ a veces, $8 \%$ pocas veces.

10.- ¿Acostumbra realizar algún deporte?

\begin{tabular}{|l|c|c|}
\hline Respuesta & Cantidad & Porcentaje \\
\hline SI & 80 & $43 \%$ \\
\hline NO & 104 & $57 \%$ \\
\hline Total & 184 & $100 \%$ \\
\hline
\end{tabular}

\section{Tabla 18}

Fuente: Escuela Héctor Lara Zambrano

Interpretación: Del total de niños afectado del (184 niños) acostumbra a realiza algún deporte el $43 \%$ Si, el $57 \%$ No

11.- ¿Con que frecuencia realiza deportes?

\begin{tabular}{|l|c|c|}
\hline Respuesta & Cantidad & Porcentaje \\
\hline Siempre & 28 & $35 \%$ \\
\hline Casi siempre & 25 & $31 \%$ \\
\hline A veces & 13 & $16 \%$ \\
\hline Pocas veces & 14 & $18 \%$ \\
\hline Total & 80 & $100 \%$ \\
\hline
\end{tabular}

Tabla 19

Fuente: Escuela Héctor Lara Zambrano

Interpretación: Del total de niños afectado del (80 niños) con qué frecuencia realiza deporte el $35 \%$ siempre, $31 \%$ casi siempre, $16 \%$ a veces, $18 \%$ pocas veces.

12 ¿Acostumbra ver televisión y utilizar videojuegos?

\begin{tabular}{|l|c|c|}
\hline Respuesta & Cantidad & Porcentaje \\
\hline SI & 164 & $89 \%$ \\
\hline NO & 20 & $11 \%$ \\
\hline Total & 184 & $100 \%$ \\
\hline
\end{tabular}

Tabla 20

Fuente: Escuela Héctor Lara Zambrano

Interpretación: Del total de niños afectado del (184 niños) acostumbra ver televisión y utilizar el 89 $\% \mathrm{Si}$, el $11 \%$ No 
12.- ¿Con que frecuencia ve televisión y utiliza los videojuegos?

\begin{tabular}{|l|c|c|}
\hline Respuesta & Cantidad & Porcentaje \\
\hline Siempre & 96 & $59 \%$ \\
\hline Casi siempre & 38 & $23 \%$ \\
\hline A veces & 23 & $14 \%$ \\
\hline Pocas veces & 7 & $4 \%$ \\
\hline Total & 164 & $100 \%$ \\
& & \\
\hline
\end{tabular}

Tabla 21

Fuente: Escuela Héctor Lara Zambrano

Interpretación: Del total de niños afectado del (164 niños) con qué frecuencia ve televisión y utiliza los videojuegos $29 \%$ siempre, $12 \%$ casi siempre, $7 \%$ a veces, $2 \%$ pocas veces.

\section{Conclusiones.}

El grupo de niños estudiados en la Escuela de educación Básica Héctor Lara Zambrano de la cuidad de Milagro, presentan una prevalencia de sobrepeso de $12 \%$ y obesidad $12 \%$ respectivamente, y una prevalencia de exceso de peso de $24 \%$, presentando un comportamiento similar con los estudios que se han realizado a nivel de América Latina, y en países en vía de desarrollo.

La prevalencia de sobrepeso (12\%) y obesidad (12\%) fue más alta en la población masculina que en la femenina (52\%) sobrepeso y (64\%) obesidad. Los niños de 7-8 años presentaron una prevalencia de $15 \%$ de sobrepeso, y $18 \%$ de obesidad

Los resultados de este estudio evidencian que los escolares consumen diariamente alimentos con alto contenido graso y calórico, y de bajo valor nutritivo $91 \%$ a diferencia del bajo consumo de frutas con porcentajes que oscilan entre $36 \%$ de los niños que las consumen diariamente.

También se puede observar, el alto consumo de azucares 51\%, .un 58\% de escolares estudiados realizan poco actividad física. En las clases de educación física, sin discriminar por su estado nutricional, todos los niños tienen una participación de la misma.

Por el contrario, un alto porcentaje $89 \%$ de los escolares con sobrepeso y obesidad dedican 1 y más de 5 horas diarias a ver televisión, al computador y a practicar videos juegos. 
Finalmente se observó que los factores que fomentan la obesidad son:

Alta ingesta de comida chatarra

\section{Sedentarismo}

Falta de actividad física.

\section{Factor Hereditario}

\section{Bibliografía.}

Álvarez, G. M. (2011). Prevalencia de sobrepeso y obesidad, y factores de riesgo, en niños de 7-12 años, en una escuela pública de Cartagena septiembre- octubre de 2010. Bogotá: Universidad Nacional de Colombia

Colchero, R. (1982). La consolidación de las fracturas, su fisiología y otros datos clínicos de importancia. Revista Médica IMSS, 21(4), 374-81.

Cordero, M. J. (2012). Estudio comparativo de la eficacia del índice de masa corporal. Nutricion Hospitalaria, 185-191.

Esquivel, F. (2010). Prevalencia de no-unión en pacientes con fractura diafisiaria de fémur tratados con clavo centromedular bloqueado en el Hospital General de Querétaro. Periodo de marzo del 2008 febrero del 2010. Tesis de especialidad, Universidad Autónoma de Querétaro, Facultad de Medicina, Mexico.

Gilardon, E. O. (2013). Sobrepeso y obesidad en niños y adolescentes.

LANAU, S. P. (2012). Educación en Obesidad infantil. 6-20.

León, A. (May de 2013). Estudio las fracturas diafisiarias de fémur con clavo intramedular bloqueado y sus resultados funcionales en el Hospital IESS Teodoro Maldonado Carbo 2011-2013. Tesis de Especialidad, Universidad de Guayaquil, Escuela de graduados, Guayaquil.

Ministerio de Salud Pública, Instituto Nacional de Estadísticas y Censos. (2013). ENSANUT. Encuesta Nacional de Salud y Nutrición, 36-37.

Morales, C. (2012). Uso de clavo endomedular retrógrado en fracturas de fémur. Patología del Aparato Locomotor, 2(1), 10-12. 
Nora Zeberio MD, Agustina Malpeli BS, María Apezteguía BS, Marta A Carballo BS, Horacio F. González MD (2012). Nutritional status of school-aged children and its relation to blood pressure. Archivos argentinos de pediatría. 111(2). 92-97

Moreno, D. M. (2012). Definición y clasificación de obesidad. 124-125.

Organisation for Economic Co-operation and Developmen (2014). Obesity Update. autor

OMS (2015). Obesidad y sobrepeso. Washington: Organización Mundial de la Salud

Von Ruden, C. (2015). Surgical treatment of ipsilateral multi-level femoral fractures. J Orthop Surg Res, 10(1), $7-11$.

Alfonso Guerra, J. P. (2013). Obesidad. La Habana, Cuba: Revista Cubana de Salud Pública.

Amelia Rodríguez Martín, J. P. (2012). La percepción del sobrepeso y la obesidad infantil por parte de los progenitores. Madrid: Revista Española de Salud Pública.

Francisco Moraga M, M. J. (2003). Tratamiento de la obesidad infantil: Factores. Santiago: Revista chilena de pediatría.

José M. Saavedra, A. M. (2012). Factores alimentarios y dietéticos asociados a la obesidad infantil: recomendaciones para su prevención antes de los dos años de vida. Lima: Revista Peruana de Medicina Experimental y Salud Pública.

Mardia G. López-Alarcón, M. R.-C. (2008). Epidemiología y genética del sobrepeso y la obesidad. Perspectiva de México en el contexto mundial. México: Boletín médico del Hospital Infantil de México.

Masud Yunes Zárraga, J. L. (2112). Obesidad infantil. México, México: Revista de Especialidades MédicoQuirúrgicas.

Ricardo, Y. R. (2012). Antropometría en el diagnóstico de pacientes obesos; una revisión. Madrid: Nutrición Hospitalaria.

Soca, P. E., \& Peña, A. N. (2009). Consecuencias de la obesidad. La Habana: ACIMED. 